\title{
Lumbosacral Interbody Fusion Using a Biportal Endoscopic Technique for Patients with Multilevel Severe Degenerative Lumbosacral Spondylosis: Technical Note and Case Presentations
}

\author{
Seok Bong Jung ${ }^{10}$ Nackhwan Kim² \\ ${ }^{1}$ Spine Center, Jinju Bon Hospital, Jinju-si, Republic of Korea \\ 2 PM\&R, Korea University Ansan Hospital, Ansan, Gyeonggi-do, \\ Republic of Korea

\begin{abstract}
Address for correspondence Nackhwan Kim, MD, PhD, PM\&R, Korea University Ansan Hospital, Ansan, Gyeonggi-do 15355, Republic of Korea (e-mail: nackhwan@gmail.com).
\end{abstract}

J Neurol Surg A Cent Eur Neurosurg

\author{
Abstract \\ Keywords \\ - lumbosacral disk \\ degeneration \\ disorders \\ - endoscopic biportal \\ surgery \\ - minimally invasive \\ spine surgery \\ - lumbar interbody \\ fusion \\ - degenerative \\ spondylosis
}

Background Open decompression and posterior interbody fusion are standard surgical interventions for multilevel degenerative lumbosacral spondylosis (DLS). Despite their clinical efficacy, intraoperative and postoperative complications have led to the demand for a minimally invasive approach. A biportal endoscopic approach is an advanced minimally invasive surgical option.

Methods The data of two patients with multilevel DLS who had undergone biportal endoscopic spine surgery (BESS) were retrospectively analyzed. Parameters such as surgical difficulty, duration of operation, blood loss, length of hospital stay, and postoperative complications were reviewed. Pain and functionality were assessed using the visual analog scale (VAS) and the Oswestry Disability Index (ODI), respectively.

Results Both patients were women and aged 75 and 73 years; they complained of back pain, claudication, pain and weakness in the lower extremities, and gait disturbance. The symptoms lasted 5 and 8 years, respectively. The multilevel BESS approach was applied bilaterally. Dissection, laminofacetectomy, decompression, excision, cage insertion, and screw implantation were performed. The operation durations were 170 and 160 minutes with blood loss of 500 and $650 \mathrm{~mL}$, respectively. Back pain, leg pain, and ODI scores significantly improved; no pseudoarthrosis or additional neurologic deficits were noted on follow-up.

Conclusions The presented BESS technique is a minimally invasive treatment option for patients with multilevel DLS, which typically requires a complicated surgical approach. Randomized controlled studies with larger sample sizes and longer follow-up periods are needed to verify the superiority of this operation. received

October 27, 2021

accepted after revision

February 15, 2022
DOI https://doi.org/

10.1055/a-1783-9999.

ISSN 2193-6315. (c) 2022. The Author(s).

This is an open access article published by Thieme under the terms of the Creative Commons Attribution-NonDerivative-NonCommercial-License, permitting copying and reproduction so long as the original work is given appropriate credit. Contents may not be used for commercial purposes, or adapted, remixed, transformed or built upon. (https://creativecommons.org/ licenses/by-nc-nd/4.0/)

Georg Thieme Verlag KG, Rüdigerstraße 14, 70469 Stuttgart, Germany 


\section{Introduction}

Degenerative lumbosacral spondylosis (DLS), with concomitant neurologic deficit and functional disabilities, is typically managed via surgery. ${ }^{1}$ Despite the clinical efficacy of this approach, intraoperative and postoperative complications have led to the demand for a minimally invasive approach. ${ }^{2}$

Biportal endoscopic spine surgery (BESS) is being increasingly applied to diskectomy and decompression surgery. ${ }^{3}$ This method adheres to the minimally invasive principle; at the same time, it is designed to realize the essence of surgical intervention by securing an increased degree of freedom for the surgeon's hand and a wide view suitable for complex bony vertebrae. Moreover, reports on its efficacy and safety are increasing. ${ }^{4}$ The surgeon can approach the anterior and posterior structures of the spine through a posterior approach with the preservation of support structures. Bilateral multilevel application is also possible. ${ }^{5}$

It may seem unreasonable to apply a minimally invasive method to the extensive surgery of multilevel degenerative spondylolisthesis. However, the advantages of BESS may be valid for sufficient decompression and careful exploration. Reports on the feasibility of BESS interbody fusion in cases of lytic spondylolisthesis and single-level degenerative spondylolisthesis have been published. ${ }^{6-8}$

Thus, here, we aim to describe a surgical technique of decompression and interbody fusion using the BESS and present clinical and radiologic results in patients with severe multilevel DLS.

\section{Methods}

This retrospective case study was conducted in compliance with the Declaration of Helsinki and approved by the institutional review board. Informed consent was obtained from each patient.

Both patients had two or more adjacent lesions. Data were gathered retrospectively and analyzed after receiving approval from the institutional review board. The data were as follows: inpatient and outpatient medical charts, nursing records, surgical records, images, and logon records from hospital information systems.

\section{Surgical Techniques}

The surgery was performed under general anesthesia. Intraoperatively, each patient's blood pressure, heart rate, electrocardiogram, oxygen saturation, and respiratory rate were monitored. The patients were placed in the prone position. Their backs were gently flexed by placing the Wilson frame under the abdomen. The frame was fixed using a soft strap on the trunk and thighs for stabilization. The lumbosacral region was exposed, while the rest of the body was covered with sterile drapes. The target site was identified under fluoroscopy, and incisions were made bilaterally based on the intervertebral disk levels for cage insertion.

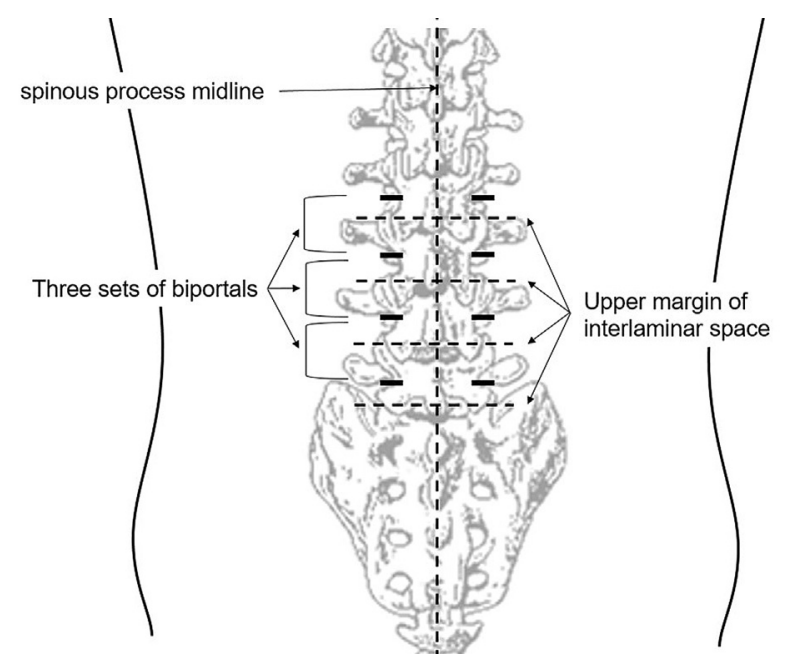

Fig. 1 Overview of biportal endoscopic spine surgery for multilevel degenerative lumbosacral spondylosis (target levels: L2/L3, L3/L4, and L4/L5). Three sets of two portals have a total of eight incision lines bilaterally, sharing an upper caudal portal and a lower cranial portal.

Step 1. Incision. Two portals were constructed as follows: the entry points were marked $1 \mathrm{~cm}$ above and $2 \mathrm{~cm}$ below the target intervertebral level (lower margin of the upper lamina) and $0.5 \mathrm{~cm}$ laterally from the spinous midline. The marked skin can be opened by $1 \mathrm{~cm}$ using the transverse incision method, providing low resistance to major movements of the inserted devices, thus reducing damage to the longitudinal muscle fibers. The cranial portal was used for continuous irrigation and endoscopy, and the caudal portal was used for the operating devices. Three sets of two portals have a total of eight incision lines bilaterally and sharing a caudal portal on the upper level and a cranial portal on the lower level ( $\mathbf{- F i g . 1} \mathbf{1}$ ).

Step 2. Approach. After muscle exfoliation using a dissector, a 30-degree endoscope with a 4-mm diameter was inserted into the target lamina. A continuous irrigation system was connected and controlled to set a pressure of $50 \mathrm{~mm} \mathrm{Hg}$. After ensuring a clear endoscopic view, an indicator was inserted into the caudal portal to identify the tip through the endoscopic view. Target points and instrument placement were confirmed through fluoroscopy.

Step 3. Laminectomy and facetectomy. The interlaminar ligament was resected 1 to $1.5 \mathrm{~cm}$ transversely. Partial laminectomy was performed using an elliptical arthroscopic burr. After inspecting the dura mater, the thickened ligamentum flavum (LF) was removed using a Kerrison punch. The neural structures of the spinal canal and foramen were inspected. The endoscope was inserted deeply to check the subarticular annulus fibrosus, and the contralateral LF and epidural space were then inspected by rotating the 30-degree endoscope (-Fig. 2). The total inferior articular process and partial superior articular process were subsequently removed using an osteotome and a chisel, thus securing the cage insertion space and root decompression. Bone fragments were picked up. 


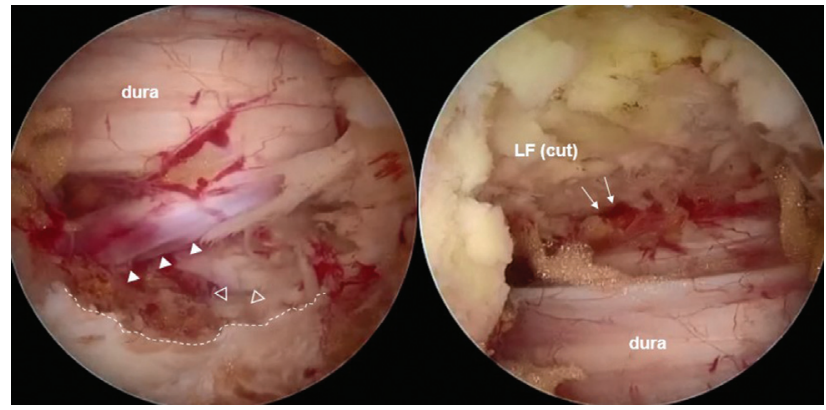

Fig. 2 Endoscopic views on the L3/L4 epidural space. Left: The dura, nerve root sleeve, and nerve root (solid arrowhead) above the annulus (open arrowhead). The dotted line is the resected lamina. Right: Contralateral epidural space. The arrow points at the foramen.

Step 4. Disk space preparation and cage insertion. Approaching the subarticular annulus fibrosus, disk materials were removed through an incised hole of the herniated annulus. The cartilage of endplate was scraped to induce bone union using a curette and a reamer. The reserved intervertebral space enabled the direct entry of the endoscope. The cage size was defined by the last reamer size. A mixture of harvestet local bony fragments with demineralized bone matrix (DBM) was packed in the cage. In this process, the neuromuscular structures were protected through a retractor.

Step 5. Contralateral decompression. Once it was confirmed, through endoscopy and radiography, that the cage placement was stable, the contralateral LF was removed and decompression was added (-Video $\mathbf{1}$ ). When the 30-degree endoscope was rotated 150 degrees axially at the level of posterior epidural space, the contralateral LF and dura were identified. The contralateral recess decompression byLF removal using the punch enabled endoscopic identification of the contralateral traversing spinal root.

Step 6. Repetition of steps 4 and 5 according to the target levels. Since the intervertebral height was restored through these operating steps, the decompression was performed form the caudal to the cranial level to prevent a traction injury of nerve roots.

Step 7. Percutaneous pedicle screw fixation. Under radiography, pedicle screws were inserted. Reduction was tried, and the presence of a listhesis was considered. The pedicle screws and the rods were locked.

Step 8. Inspection before closing. Bleeding and the integrity of spinal and neurovascular elements were closely monitored through endoscopic exploration. By seeing the dural pulsation caused by normal breathing, the degree of decompression was evaluated endoscopically. Once all the instruments were removed, a subfascial hemovac was inserted and the skin was sutured (-Fig. $\mathbf{3}$ ).

Step 9. Postoperative management. Walking with a rigid brace was allowed on the day after surgery once stable vital signs were confirmed. The patients were discharged 3 to 5 days after surgery.

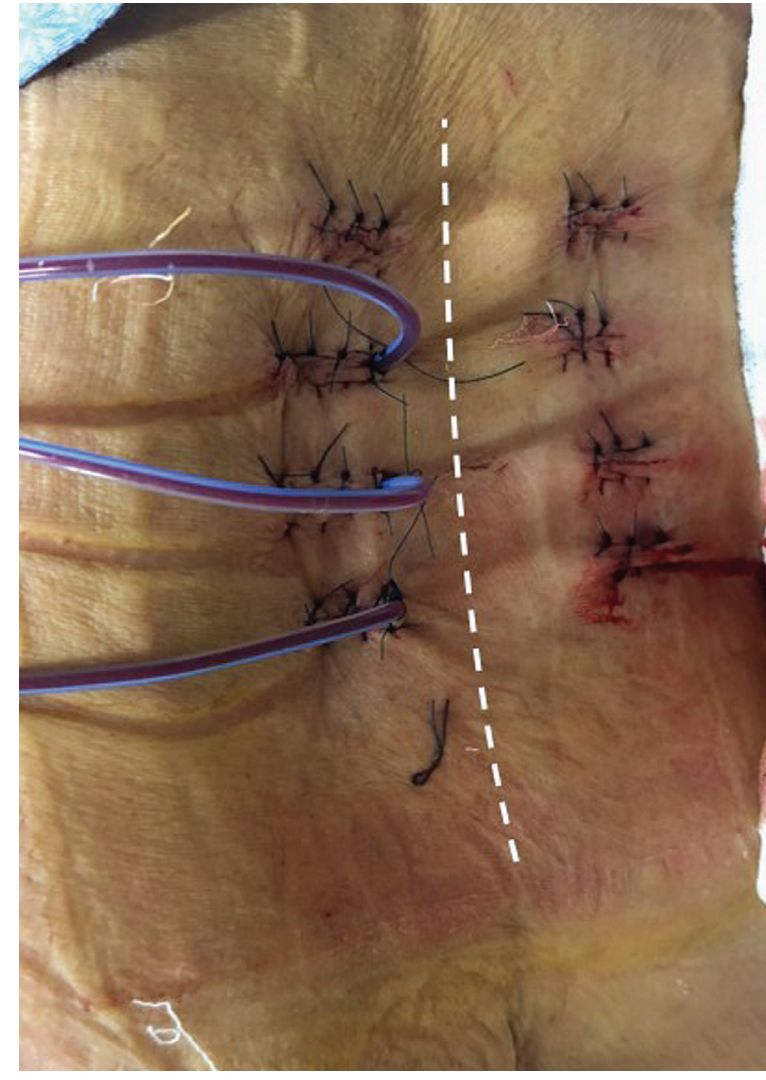

Fig. 3 The sutured skin of the portals and inserted subfascial Hemovac drain immediately after surgery. Eight incisions were placed bilaterally and the target levels were L2/L3, L3/L4, and L4/L5.

\section{Video 1}

Endoscopic view during total diskectomy and cage insertion in biportal endoscopic spinal surgery (BESS) for L4/L5 interbody fusion. Online content including video sequences viewable at: https://www.thiemeconnect.com/products/ejournals/html/10.1055/a1783-9999.

\section{Case 1}

A 75-year-old woman with no history of trauma was admitted to the hospital with chronic hip pain, continuous radicular pain, and severe claudication that had lasted 5 years. She reported that even if she walked only 10 steps, she felt calf pain and weakness, so she had to sit down and rest. She had been taking limaprost $5 \mu \mathrm{g}$ three times a day, $37.5 \mathrm{mg}$ of tramadol, and $325 \mathrm{mg}$ of acetaminophen on an irregular basis for 10 months. In the last month, the buprenorphine patch $(10 \mu \mathrm{g} / \mathrm{h}$, total $10 \mathrm{mg})$ has been used every week, but there has been no symptomatic improvement. A plain lumbosacral radiograph showed compression fractures of the third, fourth, and fifth lumbar vertebrae with severe degenerative changes in the endplates and facet joints. On 

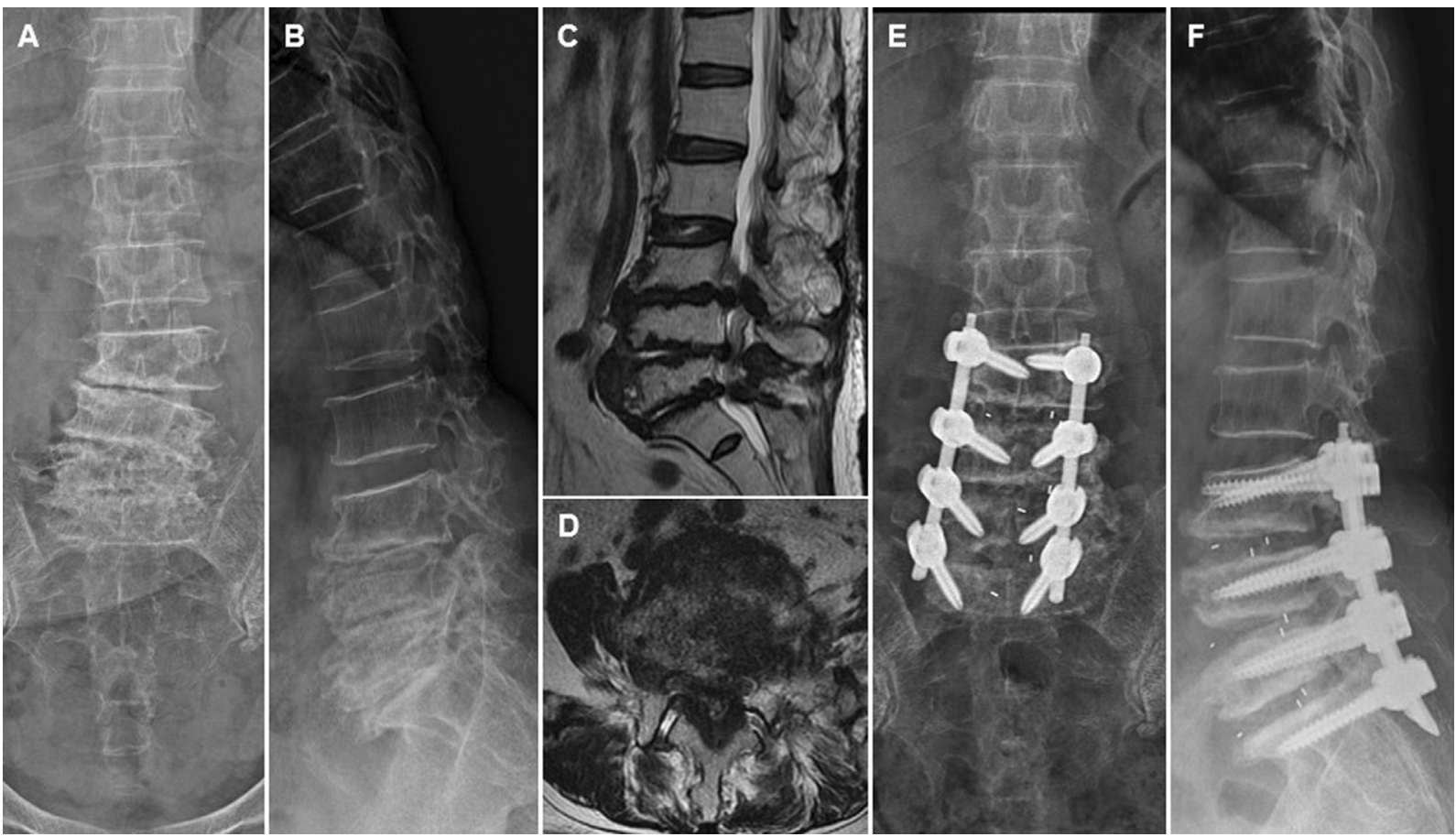

Fig. 4 Pre- and postoperative images of the case 1. (A,B) Preoperative radiographs. (C,D) Preoperative spine magnetic resonance imaging (MRI). (E,F) Radiographs 3 months after the surgery.

magnetic resonance imaging (MRI), L3/L4, L4/L5, and L5/S1 disk herniations and severe stenosis of the foramen and spinal canal were observed. Difficulty in ambulating for more than $30 \mathrm{~m}$ was noted, and the extensor and flexor muscles of both ankles showed grade 4 muscle weakness. Previous surgical history included a partial gastrectomy for gastric cancer 2 years prior. She was taking an antihypertensive medication.

She underwent biportal endoscopic decompression with a three-level interbody fusion from L3 to S1 through the unilateral biportal approach at each level. The duration of operation was 170 minutes, and blood loss was $500 \mathrm{~mL}$. Postoperative MRI and X-ray images demonstrated an improvement in the lumbar lordosis, significant decompression of the neural structures, and well-placed implants ( - Fig. 4).

Ambulation with minimal assistance was initiated on the third postoperative day. There was gradual relief of pain and claudication; 8.9 to 3.2 on the visual analogue scale (VAS) of back pain, 7.4 to 1.5 on the VAS of leg pain, and 66.7 to $19.8 \%$ on the Oswestry disability index (ODI) score 4 weeks postsurgery. Even if she walked more than $30 \mathrm{~m}$ indoor, no pain or weakness occurred. There was no evidence of pseudoarthrosis or neurologic deficits at the 1 -year follow-up. At this time, she reported that she could walk about a distance of $\sim 150 \mathrm{~m}$ without a rest. Acetaminophen was taken due to intermittent low back pain, but it did not exceed once a week.

\section{Case 2}

A 73-year-old woman presented with chronic low back pain and radicular pain and severe claudication in both legs that had lasted 8 years. The symptoms were more dominant on the right and were recently observed to worsen upon coughing. She had difficulty walking independently. She was able to move about $5 \mathrm{~m}$ under a minimal assistance, but had to rest due to right severe leg pain and bilateral legs weakness. She was taking celecoxib $100 \mathrm{mg}$ and gingko leaf extract $40 \mathrm{mg}$ twice a day for 1 year, and was also taking oxycodone $5 \mathrm{mg}$ irregularly to recent exacerbated pain. A plain lumbosacral radiograph showed degenerative malalignment of the second to fifth lumbar vertebrae with severe degenerative changes in the endplate and facet joints. On MRI, lumbar disk herniations and severe stenosis of the foramen and spinal canal were observed. The extensor muscles of both ankles showed grade 3 muscle weakness and the flexor muscles of both ankles and knees showed grade 4 muscle weakness. Electrophysiologic studies showed chronic bilateral L5 and S1 radiculopathies. She was taking antihypertensive and antiglycemic medications.

The patient underwent biportal endoscopic decompression with three-level interbody fusion from L2 to L5. The duration of operation was 160 minutes, and blood loss was $650 \mathrm{~mL}$. Her scoliosis improved partially after surgery. Sufficient decompression was achieved through increase of the intervertebral and foraminal space (-Fig. 5).

She was able to walk with a rolling walker 2 days after surgery. After 4 weeks, her symptoms gradually improved: 8.1 to 2.8 on the VAS of back pain, 7.2 to 1.9 on the VAS of leg pain, and 58.2 to $11.8 \%$ on the ODI. She showed a dramatic improvement in claudication, allowing her to walk three times $20 \mathrm{~m}$ without a rest. No pseudoarthrosis or neurologic deficits were observed, and the strength of the extensor muscles of both ankles improved to grade 4 on follow-up 18 months after surgery. At this time, she reported that she had no difficulty walking and said she could climb a 


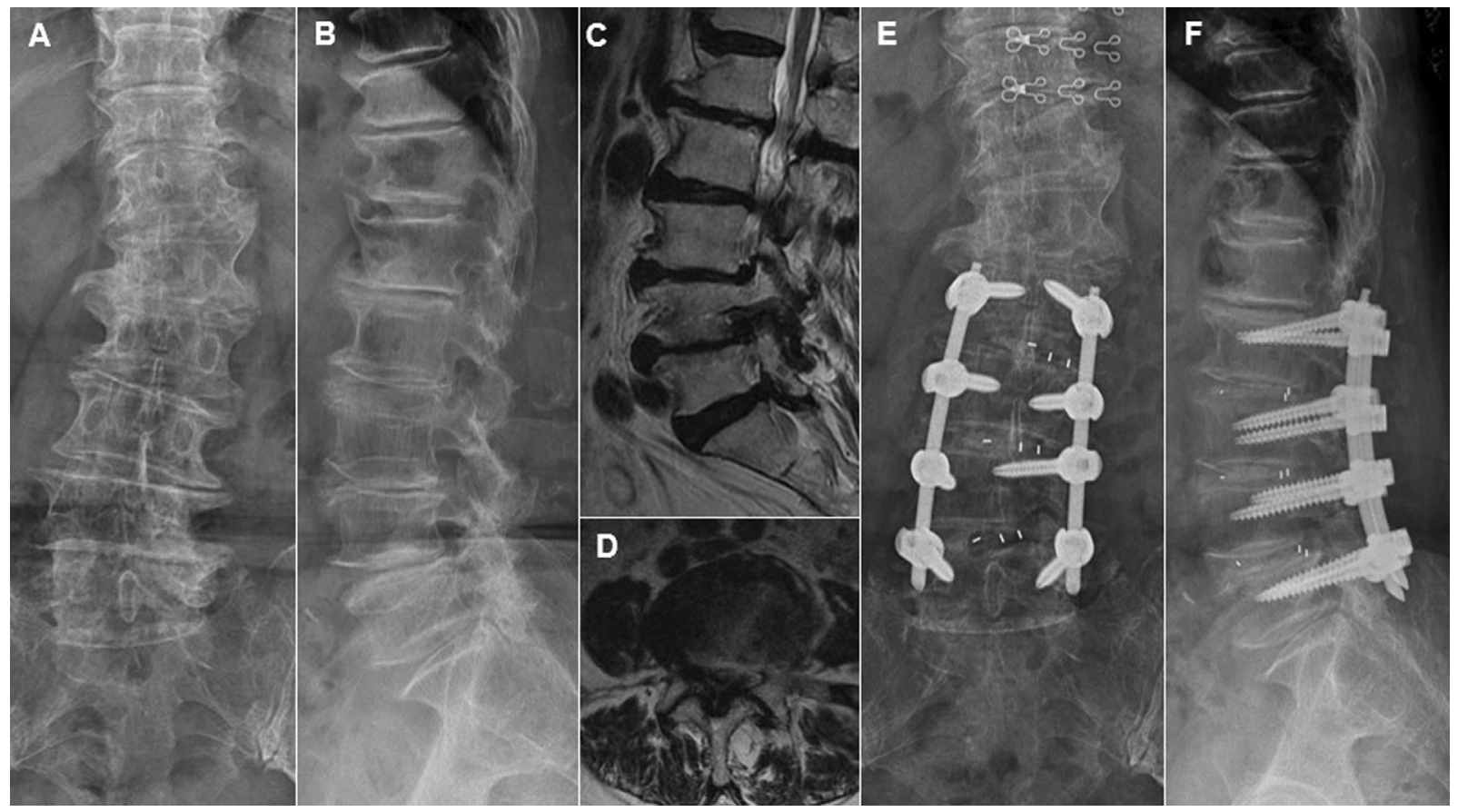

Fig. 5 Pre- and postoperative images of the case 2. (A,B) Preoperative radiographs. (C,D) Preoperative spine magnetic resonance imaging (MRI). $(\mathrm{E}, \mathrm{F})$ Radiographs 1 month after the surgery.

low-height hill. She mentioned that she often had low back pain with minimal to moderate intensity when walking continuously for more than 30 minutes outdoor, but the pain resolved after resting. She did not report taking any analgesics due to pain.

\section{Discussion}

Innovations in endoscopic instruments and surgical techniques have shifted the treatment paradigm for degenerative lumbar spine disease from open surgery to minimally invasive surgery. ${ }^{9,10}$ Various methods, depending on the approach, direction, and the structure to be removed, are available. However, the principles of endoscopic decompression and fusion maintain the concepts of open decompression with interbody fusion. Unnecessary damage should be limited based on the principle of minimal invasiveness, but the minimal intervention should be sufficient for resection, decompression, reduction and fixation. Resection of the facet joint and annulus fibrosus is unavoidable in most minimally invasive techniques. ${ }^{11-13}$

Aging initiates disk degeneration through dehydration and fragmentation of the nucleus pulposus. ${ }^{14,15}$ Degeneration affects the distribution and balance of the load on the annulus fibrosus. The accumulated load causes structural problems such as a break in annular continuity, instability of intervertebral joints, thickening of ligaments, and a decrease in the height of the intervertebral foramen; thus, a vicious cycle develops.

Surgical decompression should be considered for significant neurologic deficits. Options include decompression with extensive osteotomy, and fusion as an additional treatment. Studies on surgical outcomes were subjected to
Cochrane review, which revealed a lack of high-quality evidence. ${ }^{16}$ Case reports showed favorable surgical outcomes with low complication rates.

Reports on BESS are increasing as this method allows sufficient decompression and wide exploration as do the conventional method, but is minimally invasive. If performed on severe stenosis, favorable outcomes can be expected, BESS is applicable to multilevel disease. ${ }^{5}$ Endoscopic decompression through the posterior approach can minimize the incision site, and the direct endoscopic approach to the annulus fibrosus enables real-time confirmation of the insertion pathway of the interbody cage. In addition, it is possible to disperse the load applied to the implanted rods and screws by preserving the supportive bony structures as much as possible.

Here, we used BESS for the surgical treatment of multilevel degenerative spondylosis with concomitant radiculopathies and claudication. The two patients were at high risk for conventional surgery because of old age, and multilevel disease. Blood loss is higher in conventional surgery, which is associated with more complications especially in older patients. Selective decompression of severe intervertebral foramen stenosis was indicated to prevent the additional neurologic complications that can occur while re-establish lordosis with fusion. There were additional advantages of BESS such as the possibility to remove herniated disk material, to insert a cage and to perform annuloplasty; moreover, multilevel treatment is possible., ${ }^{3,5}$ Clinical outcomes showed remarkable pain reduction, fewer complications, and early ambulation. Daily activities improved gradually, without pseudoarthrosis or additional neurologic deficits during follow-up. There was high patient satisfaction with the surgery. Attention to the longer operation time is necessary. Constant irrigation with pressure to secure the field of 
endoscopic view during manipulation can cause neurologic damage. Evidence of increased epidural pressure during surgery has been reported. ${ }^{17}$ The intraoperative irrigation pressure should be kept below $50 \mathrm{~mm} \mathrm{Hg}$; further, if possible, neurologic monitoring is recommended.

\section{Conclusion}

More reports on the type, severity, and incidence of complications as well as the effectiveness of surgery are necessary. BESS can be applied to multilevel degenerative spondylosis. BESS provides freedom for the surgeon's hand and a sufficient operative view.

\section{Patient Consent}

The patients have consented to the submission of the case report to the journal.

\section{Funding}

This study was funded by the Korea University Medical Center Grant (K2111141).

\section{Conflict of Interest}

None declared.

\section{References}

1 Sengupta DK, Herkowitz HN. Degenerative spondylolisthesis: review of current trends and controversies. Spine 2005;30(6, Suppl):S71-S81

2 Okuda S, Miyauchi A, Oda T, Haku T, Yamamoto T, Iwasaki M. Surgical complications of posterior lumbar interbody fusion with total facetectomy in 251 patients. J Neurosurg Spine 2006;4(04): 304-309

3 Kim N, Jung SB. Percutaneous unilateral biportal endoscopic spine surgery using a 30-degree arthroscope in patients with severe lumbar spinal stenosis: a technical note. Clin Spine Surg 2019;32 (08):324-329

4 Lin GX, Huang P, Kotheeranurak V, et al. A systematic review of unilateral biportal endoscopic spinal surgery: preliminary clinical results and complications. World Neurosurg 2019;125:425-432
5 Kim N, Jung SB. Biportal endoscopic spine surgery in the treatment of multi-level spontaneous lumbar epidural hematoma: case report. J Orthop Sci 2022;27(01):288-291

6 Heo DH, Son SK, Eum JH, Park CK. Fully endoscopic lumbar interbody fusion using a percutaneous unilateral biportal endoscopic technique: technical note and preliminary clinical results. Neurosurg Focus 2017;43(02):E8

7 Quillo-Olvera J, Quillo-Reséndiz J, Quillo-Olvera D, BarreraArreola M, Kim JS. Ten-step biportal endoscopic transforaminal lumbar interbody fusion under computed tomography-based intraoperative navigation: technical report and preliminary outcomes in Mexico. Oper Neurosurg (Hagerstown) 2020;19(05): 608-618

8 Ahn Y, Youn MS, Heo DH. Endoscopic transforaminal lumbar interbody fusion: a comprehensive review. Expert Rev Med Devices 2019;16(05):373-380

9 Vaishnav AS, Othman YA, Virk SS, Gang CH, Qureshi SA. Current state of minimally invasive spine surgery. J Spine Surg 2019;5 (Suppl 1):S2-S10

10 Ahn Y. Current techniques of endoscopic decompression in spine surgery. Ann Transl Med 2019;7(Suppl 5):S169

11 He L, Feng H, Ma X, et al. Percutaneous endoscopic posterior lumbar interbody fusion for the treatment of degenerative lumbar diseases: a technical note and summary of the initial clinical outcomes. Br J Neurosurg 2021:1-6

12 Hua W, Wang B, Ke W, et al. Comparison of lumbar endoscopic unilateral laminotomy bilateral decompression and minimally invasive surgery transforaminal lumbar interbody fusion for onelevel lumbar spinal stenosis. BMC Musculoskelet Disord 2020;21 (01):785

13 Matsumoto K, Shah A, Kelkar A, et al. Biomechanical evaluation of a novel decompression surgery: transforaminal full-endoscopic lateral recess decompression (TE-LRD). N Am Spine Soc J 2020; 5:100045

14 Adams MA. Degenerative disc and vertebral disease: basic sciences. Surgery 2009;27(07):297-300

15 Gallucci M, Limbucci N, Paonessa A, Splendiani A. Degenerative disease of the spine. Neuroimaging Clin N Am 2007;17(01): 87-103

16 Machado GC, Ferreira PH, Yoo RI, et al. Surgical options for lumbar spinal stenosis. Cochrane Database Syst Rev 2016;11(11): CD012421

17 Kang T, Park SY, Lee SH, Park JH, Suh SW. Assessing changes in cervical epidural pressure during biportal endoscopic lumbar discectomy. J Neurosurg Spine 2020:1-7 